\title{
Publisher Correction: Sex-dimorphic genetic effects and novel loci for fasting glucose and insulin variability
}

Vasiliki Lagou, Reedik Mägi, Jouke- Jan Hottenga (D, Harald Grallert, John R. B. Perry, Nabila Bouatia-Naji (D, Letizia Marullo, Denis Rybin, Rick Jansen (D), Josine L. Min (B), Antigone S. Dimas, Anna Ulrich, Liudmila Zudina, Jesper R. Gådin, Longda Jiang, Alessia Faggian (D), Amélie Bonnefond (1), Joao Fadista, Maria G. Stathopoulou, Aaron Isaacs (D), Sara M. Willems (1), Pau Navarro (D, Toshiko Tanaka, Anne U. Jackson (D, May E. Montasser, Jeff R. O'Connell, Lawrence F. Bielak (D), Rebecca J. Webster, Richa Saxena (D, Jeanette M. Stafford, Beate St Pourcain, Nicholas J. Timpson, Perttu Salo, So-Youn Shin, Najaf Amin, Albert V. Smith (D, Guo Li, Niek Verweij (D, Anuj Goel D, lan Ford, Paul C. D. Johnson (1), Toby Johnson, Karen Kapur, Gudmar Thorleifsson, Rona J. Strawbridge (D), Laura J. Rasmussen-Torvik, Tõnu Esko, Evelin Mihailov, Tove Fall (D), Ross M. Fraser (D), Anubha Mahajan, Stavroula Kanoni (1), Vilmantas Giedraitis (D), Marcus E. Kleber (D), Günther Silbernagel, Julia Meyer, Martina Müller-Nurasyid (D, Andrea Ganna, Antti-Pekka Sarin, Loic Yengo, Dmitry Shungin, Jian'an Luan (1), Momoko Horikoshi, Ping An, Serena Sanna (1), Yvonne Boettcher, N. William Rayner, Ilja M. Nolte (1), Tatijana Zemunik (D), Erik van Iperen, Peter Kovacs (1), Nicholas D. Hastie, Sarah H. Wild(D, Stela McLachlan (1), Susan Campbell, Ozren Polasek, Olga Carlson, Josephine Egan (D, Wieland Kiess, Gonneke Willemsen, Johanna Kuusisto, Markku Laakso, Maria Dimitriou, Andrew A. Hicks (D, Rainer Rauramaa, Stefania Bandinelli, Barbara Thorand (D), Yongmei Liu, Iva Miljkovic, Lars Lind, Alex Doney, Markus Perola,

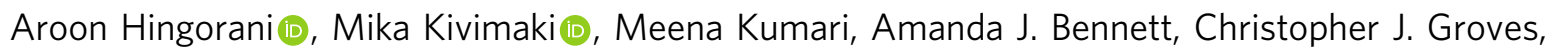
Christian Herder, Heikki A. Koistinen (10, Leena Kinnunen, Ulf de Faire, Stephan J. L. Bakker (1), Matti Uusitupa, Colin N. A. Palmer (1D, J. Wouter Jukema (D), Naveed Sattar (D), Anneli Pouta, Harold Snieder (D), Eric Boerwinkle, James S. Pankow, Patrik K. Magnusson (1), Ulrika Krus, Chiara Scapoli, Eco J. C. N. de Geus, Matthias Blüher (1), Bruce H. R. Wolffenbuttel (D), Michael A. Province, Goncalo R. Abecasis (D, James B. Meigs (D), G. Kees Hovingh, Jaana Lindström, James F. Wilson (D, Alan F. Wright, George V. Dedoussis, Stefan R. Bornstein, Peter E. H. Schwarz (D), Anke Tönjes, Bernhard R. Winkelmann, Bernhard O. Boehm (D), Winfried März, Andres Metspalu (1), Jackie F. Price, Panos Deloukas (1D, Antje Körner (1), Timo A. Lakka, Sirkka M. KeinanenKiukaanniemi, Timo E. Saaristo, Richard N. Bergman, Jaakko Tuomilehto, Nicholas J. Wareham (D,

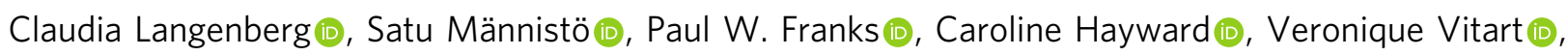
Jaakko Kaprio (1), Sophie Visvikis-Siest (10, Beverley Balkau, David Altshuler (1), Igor Rudan (1), Michael Stumvoll, Harry Campbell, Cornelia M. van Duijn (1), Christian Gieger, Thomas Illig, Luigi Ferrucci, Nancy L. Pedersen, Peter P. Pramstaller (D), Michael Boehnke (D), Timothy M. Frayling (D), Alan R. Shuldiner, Patricia A. Peyser (D), Sharon L. R. Kardia, Lyle J. Palmer, Brenda W. Penninx, Pierre Meneton, Tamara B. Harris, Gerjan Navis, Pim van der Harst (D), George Davey Smith (D), Nita G. Forouhi (D), Ruth J. F. Loos (D), Veikko Salomaa (D), 
Nicole Soranzo (1D, Dorret I. Boomsma (1D, Leif Groop (D), Tiinamaija Tuomi (1), Albert Hofman, Patricia B. Munroe (D), Vilmundur Gudnason (1), David S. Siscovick, Hugh Watkins, Cecile Lecoeur(D), Peter Vollenweider, Anders Franco-Cereceda, Per Eriksson, Marjo-Riitta Jarvelin (D, Kari Stefansson (D), Anders Hamsten, George Nicholson, Fredrik Karpe, Emmanouil T. Dermitzakis (D), Cecilia M. Lindgren (1), Mark I. McCarthy, Philippe Froguel(1), Marika A. Kaakinen (1), Valeriya Lyssenko, Richard M. Watanabe (1), Erik Ingelsson (D, Jose C. Florez, Josée Dupuis (D), Inês Barroso (D, Andrew P. Morris, Inga Prokopenko \& MetaAnalyses of Glucose and Insulin-related traits Consortium (MAGIC)*

Correction to: Nature Communications https://doi.org/10.1038/s41467-020-19366-9, published online 5 January 2021.

The original version of this Article contained an error in Fig. 2, in which panels a and b were inadvertently swapped.

This has now been corrected in the PDF and HTML versions of the Article.

Published online: 08 February 2021

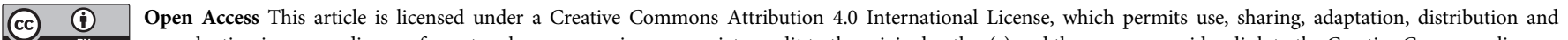
reproduction in any medium or format, as long as you give appropriate credit to the original author(s) and the source, provide a link to the Creative Commons license, and indicate if changes were made. The images or other third party material in this article are included in the article's Creative Commons license, unless indicated otherwise in a credit line to the material. If material is not included in the article's Creative Commons license and your intended use is not permitted by statutory regulation or exceeds the permitted use, you will need to obtain permission directly from the copyright holder. To view a copy of this license, visit http://creativecommons.org/licenses/by/4.0/.

(C) The Author(s) 2021 